\title{
Opinión pública y nuevas estrategias comunicativas en la industria audiovisual: el caso de Netflix y la serie Sense8
}

\author{
Francisco Javier Ruiz del Olmo ${ }^{1}$ (iD) \\ Javier Bustos Díaz ${ }^{2}$ (i)
}

\begin{abstract}
Los nuevos operadores audiovisuales muestran una creciente y mayoritaria influencia que los constituyen en una seria competencia a los canales televisivos tradicionales. Junto a ello, las redes sociales y la interactividad que los contenidos audiovisuales proporcionan con las audiencias representan un cambio de paradigma en la actividad de los públicos televisivos. En este contexto, el presente artículo analiza, mediante un estudio de caso, la situación de opinión pública y crisis comunicativa que se produjo a partir de la cancelación de contenidos (la serie Sense8, 2017, producida por Netflix) y la repercusión en redes sociales (Twitter). Entre sus conclusiones se apunta a una novedosa estrategia por parte de esta nueva compañía audiovisual para responder e integrar en su discurso y prácticas productivas las demandas de sus audiencias.
\end{abstract}

Palabras clave: comunicación en crisis; operadores audiovisuales; Netflix; Twitter; Sense8

\section{Introducción}

En los últimos años se está asistiendo de forma global a una profunda transformación del sistema de uso y consumo audiovisual, singularmente el producido en el medio televisivo. La televisión tradicional, el medio de comunicación más influyente y poderoso del siglo $\mathrm{XX}$, ha visto redefinido su papel, a partir de la aparición de diferentes medios audiovisuales y también de nuevos operadores, alejados de las lógicas emisoras y comunicativas de décadas pasadas, y donde la red y el poder de las audiencias son decisivos. En ese sentido, la opinión pública, a partir de segmentos organizados de las audiencias, ha adquirido un papel decisivo para el devenir de las empresas de los medios

\footnotetext{
1 Departamento de Comunicación Audiovisual y Publicidad, Universidad de Málaga. Málaga (Andaluzia), Espanha. E-mail: <fjruiz@uma.es>.

2 Departamento de Comunicación Audiovisual, Universidad de Málaga. Málaga (Andaluzia), Espanha.

E-mail: <bustos090@gmail.com>.
} 
(Valenzuela, Puente y Flores, 2017). En ese sentido aquí juega un papel esencial la teoría de la denominada agenda setting, formulada por McCombs y Shaw (1972). Concretamente se refiere a que la agenda mediática establece la agenda del público, es decir, el público recurre a las pistas de relevancia que le ofrecen los medios de comunicación de masas para organizar su propia agenda y decidir cuáles son los temas más importantes en la consideración pública (McCombs, 1996); la agenda de los medios de información, la agenda mediática, se convierte en la agenda pública, y a su vez en opinión pública (Rubio Ferreres, 2009).

En ese sentido ya Lippmann (2003) definía en su obra Opinión pública la formación de un modelo de opinión pública dependiendo de los medios de comunicación. Demostró que los medios informativos, esas ventanas abiertas al inmenso mundo que queda más allá de nuestra experiencia directa, determinan los mapas cognitivos que nos hacemos de él (Rubio Ferreres, 2009); la influencia de los medios sobre la sociedad, que, en mayor o menor medida, se ve determinada por la imagen que representa los medios sobre sí misma, es decir, "nuestra imagen del mundo" (McCombs, 1996, p. 17).

Asimismo, otros autores se alinean con la perspectiva de Habermas, que define el espacio público como "un ámbito de nuestra vida social, en el que se puede construir algo así como opinión pública. La entrada está fundamentalmente abierta a todos los ciudadanos" (1973, p. 61). Del mismo modo, Rubio Ferreres (2009) concreta que la opinión pública es un fenómeno comunicativo y psicosocial que depende del contexto histórico y sociocultural; está íntimamente relacionado con el tipo de sociedad, la cultura imperante y los intereses políticos del momento. Completando estos aspectos, encontramos algunos elementos que aporta el sociólogo británico Raymond (1998), quien sostiene que en un momento histórico concreto podemos encontrar tres tipos de cultura: residual, dominante y emergente. En estos momentos, a menudo nuestra atención se centra en las instituciones residuales 0 , al menos, en las que tienen problemas para mantener su posición hegemónica, olvidamos las dominantes y apenas intuimos las emergentes. Así pues, la democracia está siendo transformada a medida que evolucionan sus cimientos sociales, culturales y políticos (Dahlgren, 2012, p. 179); en este proceso juegan un papel esencial los medios de comunicación como de forma cada vez más rotunda las redes sociales.

Junto a esto, el actual paradigma de consumo audiovisual es la consecuencia de la combinación de factores de los medios viejos y los nuevos, que han sumado diversos productos comunicativos en un único canal: Internet (Izquierdo-Castillo, 2015). Así se sintetiza la práctica comunicativa en la que la televisión ya no se reproduce sólo en el televisor; en este sentido, Internet y la telefonía móvil han aparecido como los nuevos distribuidores de contenido audiovisual (Ruano, 2009) de tal forma que algunos medios públicos se han visto incapaces o no han sabido adaptarse con éxito a entornos online como la web o el móvil (Ruiz del Olmo y Belmonte Jiménez, 2014) donde los medios de comunicación clásicos no acaban de encontrar la fórmula (Gómez-Uribe, 2015). 
La televisión sigue siendo, con todo, el eje central del consumo audiovisual, pero cada vez más la producción y el consumo selectivo vía Internet, junto con el elemento generacional, son claves fundamentales para definir el modo en el que los operadores definen sus programaciones y sus ofertas de canales y contenidos. Esta lógica lleva, por ejemplo, a la aparición de espacios dirigidos específicamente a públicos o nichos socioeconómicos o también de edades determinadas, a partir de etiquetas o conceptos tomados como referentes para identificar a las diferentes generaciones (Woods, 2016). Frente a la tradicional propuesta televisiva, de consumo de imágenes en el electrodoméstico televisual, la aparición de nuevos operadores fideliza de forma más peculiar e intensa a los públicos con productos (mayoritariamente ficciones) con formas audiovisuales y sobre todo temáticas que no tendrían cabida en la televisión tradicional. Así, el concepto de televisión ha sido reemplazado en las nuevas políticas europeas por el de servicios audiovisuales, explica Campos-Freire (2015), de modo que los servicios audiovisuales están fácilmente a disposición de la audiencia. Se han roto ciertos cánones de la televisión tradicional, afectando a elementos tan importantes como la parrilla televisiva.

Esas transformaciones motivan precisamente el objetivo de este artículo, que indaga cómo las comunidades virtuales vinculadas a los productos de ficción construyen un poder autónomo, conformando una opinión pública mediática que se viraliza y obliga a las corporaciones audiovisuales a tenerlas en cuenta. Específicamente se analiza la forma en la que en una red social como Twitter se conforman comunidades influyentes capaces de generar opinión pública y de influir en las productoras audiovisuales. Se utiliza el estudio de caso la serie Sense8 (2017), una de las principales propuestas de la parrilla de producción propia de Netflix en su etapa de expansión internacional, y se detallan las dinámicas de opinión que motivaron tanto el aviso de cancelación del producto por parte de Netflix, así como su posterior rectificación.

Para llevar a cabo ese objetivo se ha realizado un estudio mixto, cualitativo y cuantitativo, sobre esas comunidades virtuales en la red social Twitter entre el 1 y el 29 de junio de 2017, fechas de inicio y conclusión de la crisis comunicativa; se han utilizado herramientas de investigación básica documental y trabajo de campo de observación y análisis de las comunidades virtuales seguidoras de la serie en Twitter, a partir de análisis de textos de las comunicaciones de usuarios, creadores de la serie y la productora, más un procesado cuantitativo de esa interacción.

Antes de la aplicación del método para la consecución del anterior objetivo propuesto, parece necesario referirse al novedoso contexto audiovisual y de audiencias al que nos enfrentamos, para entender el poder de generación de opinión pública de esas comunidades. En la actualidad, el consumidor de contenidos busca y selecciona ficción o información en productos audiovisuales que le entretengan en el momento preciso o inmediato que desee (Campos-Freire, 2015). En este aspecto son significantes dos ideas: de una parte, la audiencia cada vez reclama una mayor participación y un uso "a la carta" 
del material audiovisual, y de otra, el mercado audiovisual actual tiende cada vez más a centrarse en los contenidos, tal y como advirtiera Ruano (2009), tendencia que ha ido incrementándose en los últimos años. Dentro de las nuevas formas de consumo de contenido audiovisual, en la actualidad la actividad e interactividad se tienen muy en cuenta a la hora de entender y analizar una serie de televisión, elementos de su estructura y contenido que Jenkins (2003) definiera en el concepto de transmedia storytelling según el cual una narrativa se dispersa en varias plataformas o canales, siendo éstas independientes entre sí, o lo que es lo mismo, no es necesario leer el libro para entender la película y viceversa. Es en este proceso donde Jenkins (2003) posteriormente explica que una de las características principales de este fenómeno es la participación de las audiencias.

Por ejemplo, en España, tal y como recoge el Panel de Hogares de la Comisión Nacional de los Mercados y la Competencia - CNMC (2017), el tiempo dedicado por los españoles a visualizar contenidos audiovisuales en el televisor y en otras pantallas empieza a equilibrarse. En datos, el informe elaborado por la CNMC explica que en España se consumen casi tres horas en la pantalla de TV, situándose en dos horas y media el tiempo que se dedica a consumir contenido audiovisual en ordenador, tablets o smartphones. Estas son tendencias similares u homogéneas en otros países desarrollados.

Por tanto, y como consecuencia de lo apuntado anteriormente, en la actualidad "se sustituye el flujo unidireccional de los contenidos por una corriente circular y continua, que facilita la personalización del consumo por parte de la audiencia" (González-Neira y Quintas-Froufe, 2016, p. 28). Se sustancia en definitiva que esta transformación ha cambiado la forma de entender y actuar de las audiencias, cuyo poder es presagiado por audiencias específicas y segmentadas:

La televisión tradicional ha privilegiado por años a las grandes audiencias. A contracorriente, una nueva forma de entender el entretenimiento, en beneficio de los públicos minoritarios, empieza a operar en Internet. El éxito de este modelo anuncia quizá la televisión por venir (Wu, 2014, p. 10).

Significativamente, sin embargo, el aumento de la oferta audiovisual, interactiva y a la carta ha elevado e incrementado el consumo audiovisual. De nuevo en España, como ejemplo, y de forma similar en otros países europeos, el estudio de los hábitos de recepción televisiva de los públicos se ha incrementado significativamente en los últimos años. Respecto a la televisión y al consumo en red, numerosos análisis en ese sentido, y también diversos en cuanto a las técnicas metodológicas utilizadas, recogen la transformación derivada de la expansión y la generalización del uso de Internet (Claes y Deltell, 2015). Como conclusiones comunes se atestigua que los grupos de edad de los jóvenes y los adolescentes se alejan cada vez más del consumo televisivo tradicional, al no satisfacer sus demandas con la misma intensidad y variedad temática y formal que la oferta 
audiovisual digital (Bernal Triviño, 2009; López Vidales, González Aldea y Medina de la Villa, 2011).

Campos-Freire (2015), así como otros autores, apunta que "Las redes sociales digitales son un nuevo paradigma del ecosistema actual de medios" (p. 144). La digitalización y sus consecuencias parecen ser un hecho cada vez más evidente que los pilares básicos del fenómeno televisión y redes sociales son interactividad, convergencia y multimedia. Justamente sobre esas características se asienta la formación y consolidación de opinión pública mediática, que, por la naturaleza segmentada de las audiencias, es necesariamente especializada y singular.

\section{Gráfico 1}

Evolución de la penetración y uso de redes sociales en España (\%)

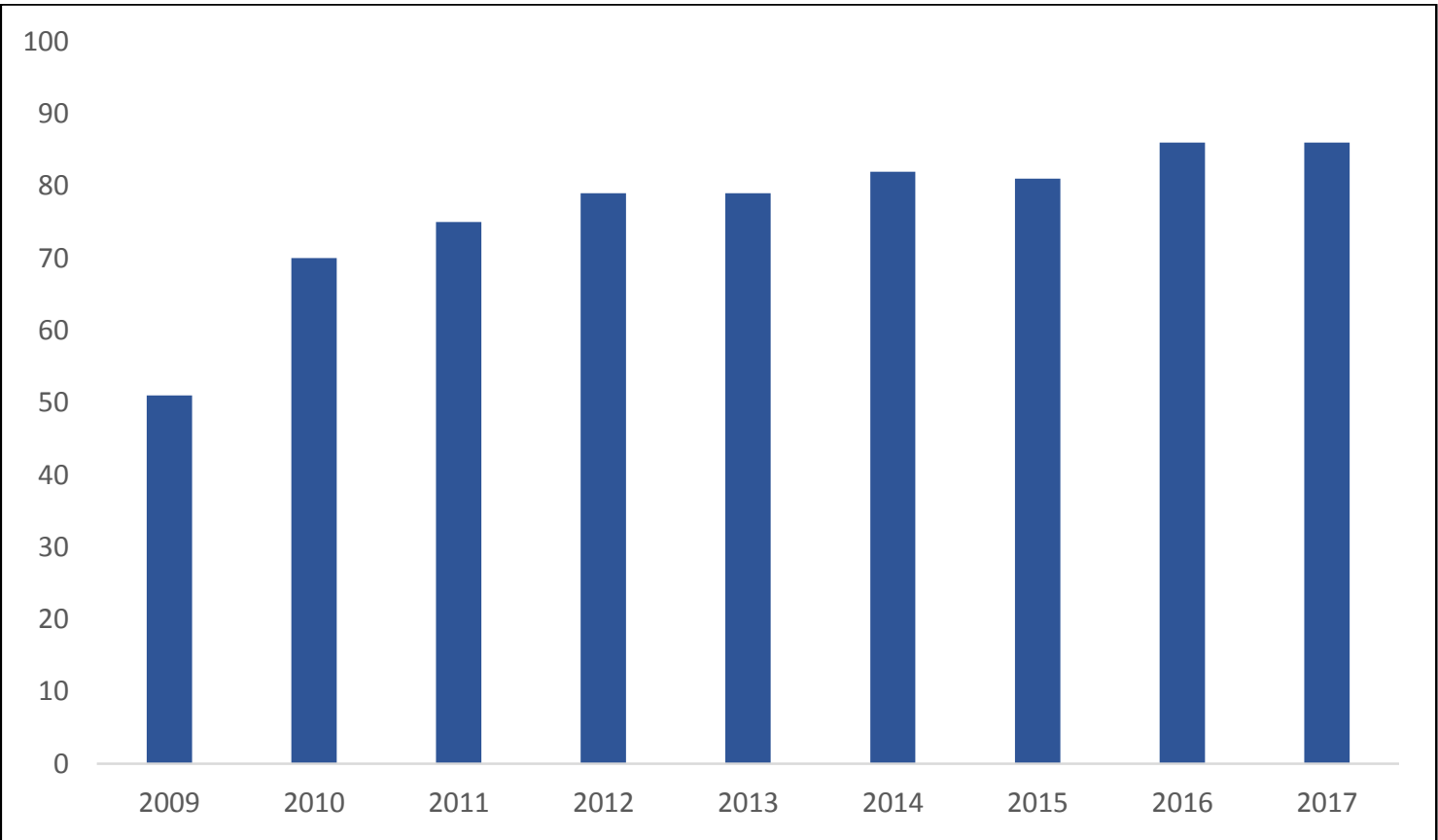

Fuente: IAB-Spain (2017).

Netflix, la televisión de pago y el constante escrutinio de la audiencia

En una sociedad multipantalla como la actual, la televisión mantiene un papel esencial dentro del nuevo escenario comunicativo (Rodríguez Rossell y Melgarejo Moreno, 2012). Según el informe "La sociedad de la información en España 2016" (AIMC), en datos absolutos la televisión $(99,3 \%)$ y el teléfono móvil $(96,7 \%)$ son las tecnologías de la comunicación con mayor presencia en los hogares españoles.

Paralelamente, el "Estudio general de medios" (AIMC) señala que el $80 \%$ de los jóvenes, aunque con reducción de tiempo y sobre todo cambio de prácticas, continúan 
consumiendo ficciones en la pantalla televisiva tradicional frente a otras plataformas como Youtube, Vimeo u otras. Estas mutaciones en las costumbres televisivas confluyen con el protagonismo de las redes sociales como medio simultáneo e instantáneo para el intercambio de comentarios sobre las emisiones entre jóvenes, como ocurre en el caso de Twitter. La pujanza de dichas prácticas para interactuar, por ejemplo, a través de los contenidos de ficción o de entretenimiento les hace sentir parte de un colectivo más grande (Wohn y $\mathrm{Na}, 2011$ ), o conectar con personajes famosos sin intermediación (Lacalle, 2011).

Todos estos fenómenos de interacción y autoafirmación identitaria con narraciones y personajes han generado nuevas estrategias de fidelización de los operadores televisivos, como es el caso de Netflix, del que a continuación nos ocuparemos, a la vez que transforman después a los operadores tradicionales a la hora de intentar captar al público de menor edad, apostando por iniciativas ligadas a narrativas transmedia. Al contexto descrito debe sumarse el éxito reciente de la gran ficción televisiva y especialmente de las series estadounidenses, descrito en diversos trabajos (Gillan, 2011).

De lo expuesto anteriormente se extraen dos ideas esenciales a la hora de entender y analizar el futuro, ya muy presente ciertamente, del modelo televisivo: multiplataforma y distribución de contenidos a la carta, especialmente ficción en forma de series o filmes.

La sociedad actual y, sobre todo, el avance tecnológico de ésta permite al usuario consumir contenidos en cualquier lugar vía streaming. Se entiende como aplicación de streaming aquella en la cual el receptor reproduce el contenido recibido (audio, video etc.) conforme va llegando sin necesidad de descargarlo completamente antes (Montoya, 2003). Entre las diversas plataformas que permiten al usuario consumir contenido acorde a estas características, destaca Netflix por su volumen, temática y difusión. Como empresa, Netflix inició su camino en 1997 como un servicio de alquiler de DVD en línea.

Con la normalización de Internet, Netflix se reinventó como una plataforma de distribución de contenido online (Heredia Ruiz, 2017). En la actualidad, tal y como explican Adhikari et al. (2015), la plataforma de video streaming funciona acorde a cuatro componentes esenciales: centro de datos de Netflix, nube de Amazon, CDN y jugadores. Esencialmente, y para comprender el funcionamiento del modelo Netflix, así como su evolución, se sintetizan las conclusiones a las que llega Jenner:

Netflix siempre ha estado más asociado con el medio de los DVD e Internet (como su nombre ya lo sugiere) en lugar de la transmisión y la programación original. No es de extrañar que, junto con las cadenas de televisión que ofrecen servicios multiplataforma, el negocio de los servicios de alquiler de videos ahora sea el que lucha por dominar el negocio de los servicios de VOD, reinventándose para una nueva era en la distribución de películas y televisión (Jenner, 2016, p. 261). 
Gráfico 2

Representación de la penetración de la TV de pago en España (\%)

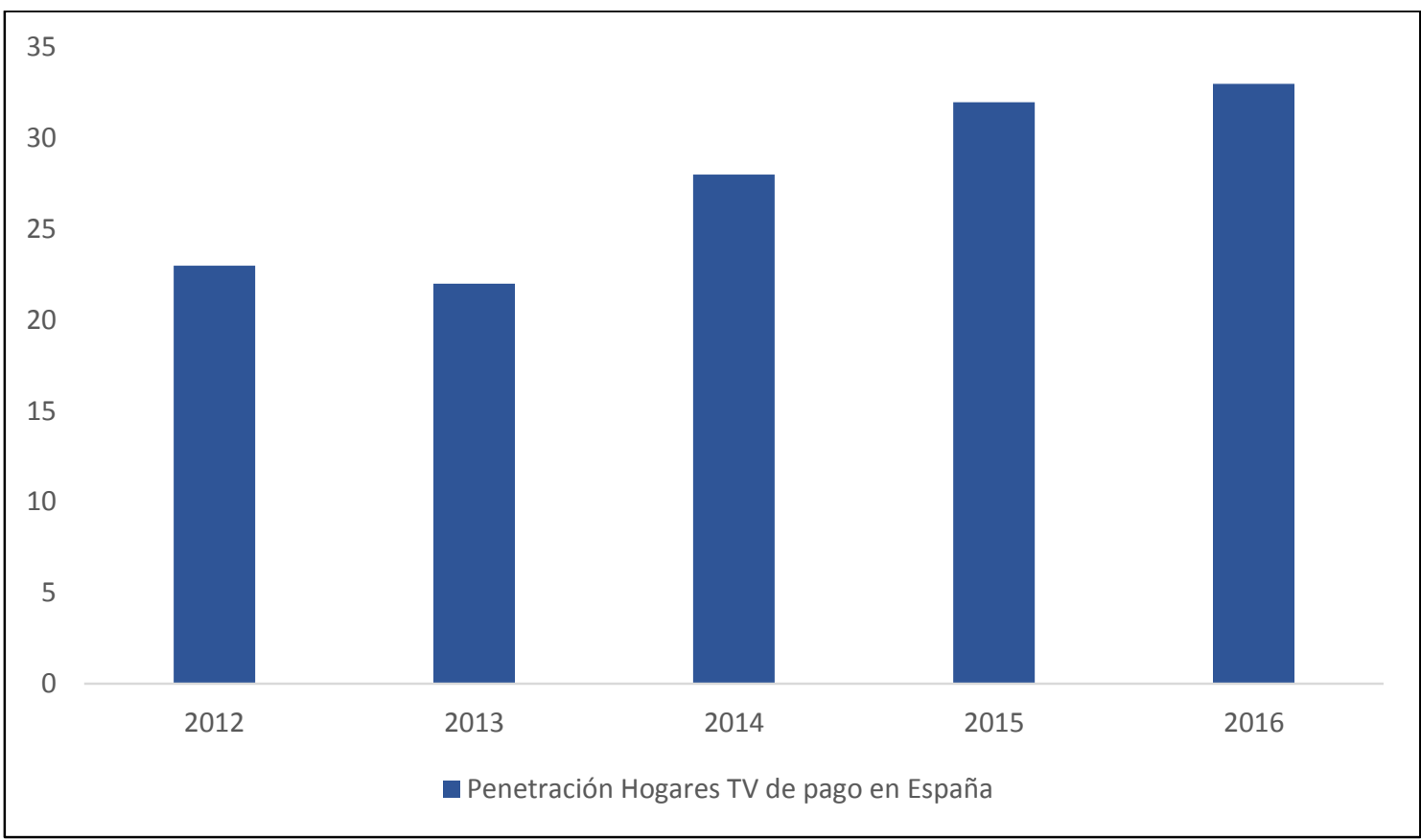

Fuente: Informe Conecta (2018).

La serie Sense8 como generadora de opinión y de corrientes empáticas en la audiencia

Más allá de un modelo tecnológico avanzado y radicalmente diferente a la televisión convencional, mediante streaming o flujo multimedia bajo demanda por Internet de banda ancha (Heredia Ruiz, 2017), las nuevas plataformas televisivas de producción y distribución de productos audiovisuales han buscado estrategias de conexión emocional con sus públicos, clientes y suscriptores (Greer y Ferguson, 2011). En ese sentido, el caso de Netflix es paradigmático; la compañía californiana, convertida ya en un gigante del entretenimiento popular doméstico con alrededor de 90 millones de suscriptores en todo el mundo; y ello a pesar de su juventud, ya que inició su expansión internacional en el año 2010. A través de una estrategia comunicativa sólida, audaz y en muchos casos polémica por su apelación directa o irónica a la actualidad, especialmente en redes como Twitter y Youtube, siempre perfectamente empática con sus públicos, ha ido construyendo una imagen de marca ligada a la ficción seriada de calidad y ampliando progresivamente el catálogo de contenidos (Wu, 2014).

En su crecimiento y consolidación internacional la compañía apostó inicialmente por usuarios jóvenes, urbanos, con cultura audiovisual avanzada y elevado consumo mediático, audiovisual en redes. Y ello no solo en cuanto a los productos disponibles sino también en cuanto a la estrategia comunicativa comercial y de márquetin en las redes 
sociales y en la prensa. Todo ello a través de la producción, promoción y difusión de historias entendibles transculturalmente, atendiendo a los flujos de narrativas ficcionales internacionales. Para ese público se crearon productos singulares, base para la expansión hacia contenidos y públicos más adultos y variados. En esa estrategia encajan series de la compañía como Sense8 (2015), ideada por las prestigiosas creadoras cinematográficas Lana y Lilly Wachowski y por Michael Straczynski. Esta ficción fue, como se ha señalado, una de las apuestas más arriesgadas de Netflix, tanto visual como temáticamente, cuando se estrenó en junio de 2015. Las hermanas Wachowski filmaron por todo el mundo su particular fresco de amor a la empatía, la humanidad y la diversidad, ante las dificultades personales y las conspiraciones globales.

La serie Sense8 presenta una historia que involucra a ocho personas repartidas por el mundo que comparten sensaciones, recuerdos y habilidades; ocho personajes que sienten, se alegran y sufren como uno solo, en un particular género de ciencia ficción realista que evoluciona progresivamente hacia el realismo descriptivo, mágico, barroco en ocasiones y preciosista; así nos topamos con una científica de Bombay (Tina Desai), un conductor de autobús en Nairobi (Aml Ameen), un ladrón de cajas fuertes berlinés (Max Riemel), una mujer de negocios de Seúl (Doona Bae), un actor mexicano (Miguel Ángel Silvestre), una DJ islandesa afincada en Londres (Tuppence Middleton), un policía de Chicago (Brian J. Smith) y una blogger y hacker política de San Francisco (Jamie Clayton). Para ello se utilizan localizaciones reales, con distintos equipos de producción por todo el mundo, con rodajes en Alemania, Islandia, Kenia, distintos puntos de los Estados Unidos, México, Corea del Sur, India y el Reino Unido.

A pesar de sus diferencias geográficas, sociales y culturales, estos ocho personajes constituyen en la trama de la serie un singular y especial tipo de ser humano, que se vincula a través del "psycellium", un sistema nervioso psíquico que permite interactuar y compartir experiencias corporales y emocionales, además de participar física y mentalmente en el devenir cotidiano de los demás. A través de una realización audiovisual muy libre y arriesgada, de amplia libertad narrativa y rompiendo las convenciones espaciotemporales de la narración clásica cinematográfica, cada uno de los personajes se traslada de facto al escenario donde se encuentra el otro, compartiendo con él la acción en la lucha contra el mal o en apoyo sentimental. En todo caso, como supranarrativa, las autoras enfatizan que sólo juntos, como participantes de una comunidad enfática, podríamos los humanos hacer frente tanto a nuestras dudas sobre la existencia y la identidad, así como superar los peligros que emanan de las conspiraciones transnacionales.

Por todo ello, y en consonancia con las temáticas que aborda, en términos de forma, Sense8 es un texto que habita en un transuniverso: transgénero, transsubjetivo y transfísico. Y también es, por eso mismo, un texto transmedia, en la medida en que Sense8 participa de lo que Jenkins, Ford e Green (2013) describen como "construcción global" o la creación de narrativas aumentadas con mundos de historia inmersivos complejos, que trascienden los límites del espectáculo en sí. 
Además, es relevante la temática de la identidad sexual, en parte a través de la influencia de la propia condición transexual de una de las creadoras de la serie, Lana Wachowski; Sense8 se muestra también como una serie que ofrece esas representaciones televisuales como una categoría de identidad cada vez más transnacional y translingual. No es sólo el primer programa de televisión que presenta un personaje transgénero escrito/dirigido por creadores trans y actuado por un artista trans, sino que también intenta visualizar un imaginario global de maneras nuevas de identidad. Para Anaz (2017) son, sin embargo, representaciones arquetípicas de la diversidad: construcciones de representaciones de la diversidad contemporánea en sus diferentes esferas, desde la étnica (cultural, racial y religiosa) hasta la sexual, así como la socioeconómica. Autores como Keegan (2016) analizan Sense8 como un texto que representa y replica simultáneamente las condiciones de la globalidad hipermoderna, discutiendo cómo las representaciones de Sense 8 se tensan contra $y$, sin embargo, reproducen parcialmente las condiciones del estereotipo. Con todo, Sense 8 enfatiza que aquello que nos une como seres humanos es más potente y universal que las diferencias culturales, raciales, religiosas, sexuales 0 nacionales.

Financieramente las autoras habían trabajado en el cine de alto presupuesto (con filmes como Matrix o Cloud Atlas) y en este caso Netflix mantuvo esos estándares. A pesar de su alto coste de producción, la rentabilidad de la serie iba ligada justamente a la empatía en ese público inicial, "evangelizador", de la plataforma. La primera situación de crisis con este público que Netflix tuvo que afrontar se produjo precisamente a partir de la cancelación y posterior reproducción de la mencionada serie.

Así comunicaba Cindy Holland, vicepresidenta de contenido de la compañía, la cancelación de la serie: "Después de 23 episodios, 16 ciudades y 13 países, la historia de Sense8 está llegando a su fin. Es lo que nosotros y los fans soñamos que sería: atrevida, emotiva, impresionante, explosiva y totalmente inolvidable. Nunca ha habido un espectáculo más verdaderamente global con un elenco igualmente diverso e internacional y el equipo, que sólo es reflejado por la comunidad de los fans profundamente apasionados de todo el mundo. Damos las gracias a Lana, Lily, Joe y Grant por su visión, y todo el elenco y el equipo por su habilidad y compromiso" (The Hollywood Reporter, 2017). Todo ello inicia un proceso de activación de audiencias que ya venían teniendo un papel activo en redes sobre la serie, y una reconfiguración del fenómeno fan, generándose un estado de opinión pública (especializada y singular, eso sí) sobre la que Netflix tuvo que retocar su estrategia de contenidos y también de comunicación con esta parte sensible de sus públicos.

\section{Objetivos}

A través de un estudio de caso especialmente significativo, el presente artículo se desarrolla en torno a la hipótesis que, en la actualidad, los nuevos productores 
audiovisuales están expuestos a las demandas y quejas de los usuarios a través de las redes sociales y que éstas generan una opinión pública compartida, fuertemente imbricada en los fenómenos de identificación colectiva. El objetivo general del artículo es identificar la forma en la que las redes sociales, singularmente Twitter, agrupan a los seguidores de determinado contenido audiovisual y son capaces de generar opinión pública y materializar de algún modo en un cambio de rumbo dentro de la propia dinámica empresarial de las productoras audiovisuales, en este caso, de Netflix. Así, nuestra propuesta se ha centrado en estudiar un caso concreto, la serie Sense8 (2017), una de las principales propuestas de la parrilla de producción propia de Netflix y observar los efectos derivados del aviso de cancelación por parte de la compañía, así como su posterior reprogramación.

Esto nos llevará a identificar nuevas formas de interacción comunicativa entre la empresa y el usuario. Esto es, y como es sabido, el movimiento derivado de los usuarios en Twitter llevó a Netflix a anunciar un capítulo final que diera respuesta a las peticiones de los fans tras el anuncio de cancelación de la serie. Como consecuencia inevitable, Netflix ha creado un precedente novedoso de cara a futuras cancelaciones que puedan derivar de la falta de audiencia o de los costes elevados que pueda producir una serie.

En definitiva, en el cambiante contexto audiovisual actual, este artículo busca definir los rasgos de las políticas comunicativas en Internet, generadoras de opinión pública y especialmente en la red social Twitter, de los nuevos operadores televisivos y audiovisuales. $Y$ conocer en concreto como se producen las estrategias comunicativas de generación de opinión pública de las audiencias concretas o especializadas. Ambas cuestiones pueden presentar una estrecha interconexión. La investigación apunta también al conocimiento de las estrategias comunicativas de expansión internacional y captación de nuevos públicos de uno de esos nuevos operadores, vía streaming y bajo demanda, Netflix, cuyo objetivo sería la captación de nuevos públicos. Igualmente, el artículo trata de definir el papel de las audiencias en la red social Twitter y su interacción con la serie y la propia compañía.

\section{Metodología}

Para el tratamiento metodológico de los objetivos previos, hemos hecho uso de una metodología multidisciplinar. En ese sentido el presente artículo realiza un estudio mixto, cualitativo y cuantitativo, sobre las estrategias de la compañía y su política de comunicación en la red social Twitter a partir de este episodio, en el periodo comprendido entre el 1 y el 29 de junio de 2017, fechas de inicio y resolución de la crisis.

Ello ha implicado tanto elementos y herramientas de investigación básica documental y bibliográfica como trabajo de campo de observación y análisis en plataformas, comunidades y redes virtuales, específicamente la red social Twitter. En la ejecución del análisis se ha optado por utilizar dos de las herramientas metodológicas que, aplicadas en cada uno de los objetivos para analizar los diferentes aspectos de un mismo 
objeto de estudio, no solo aportan perspectivas diferentes, sino que unas actúan sobre las otras de forma dialéctica, arrojando resultados innovadores y con mayor capacidad de incidencia, tanto en términos de representatividad, impacto científico, así como también de extrapolación a otros casos del nuevo panorama televisivo.

Las herramientas metodológicas que se han implementado en la presente investigación, motivadas por los objetivos propuestos anteriormente, son en síntesis las siguientes: Análisis de Textos (AT), cualitativo, referidos a las comunicaciones e interactividad de usuarios, creadores de la serie Sense8 y la propia compañía; Tratamiento Cuantitativo (TC) de esa interacción; y Estudio de Caso (EC). Se cuenta aquí con la premisa de que el estudio de caso (Yin, 2009) combinado con el análisis cualitativo y su posterior categorización y análisis es extrapolable a la resolución de crisis, con ayuda de las redes sociales y Twitter a través de estrategias comunicativas adecuadas.

\section{Resultados}

Como se ha señalado anteriormente, a partir del 1 de junio de 2017, Netflix anuncia que la serie Sense 8 iba a ser cancelada. Este hecho produce un impacto inmediato en las redes sociales $\mathrm{y}$, sobre todo, dentro de la comunidad de usuarios generada en torno a la serie y a su comunidad mediática en redes. Desde ese momento, se genera todo un fenómeno de opinión en esas mismas redes que tiene como único objetivo la producción de una temporada final de la serie 0 , al menos, de un episodio final que cierra la trama argumental de la misma.

Twitter es la red social elegida por los usuarios para comenzar una lucha o interacción contra Netflix, que encuentra en Change.org su base principal para recaudar firmas y lograr el objetivo. Bajo los hashtags \#RenewSense8 y \#BringBackSense8 se organiza toda una comunidad formada por los early adopters de la compañía, públicos vitales en la expansión internacional de la plataforma, que pone inquieta a la misma toda vez. Estos primeros adoptantes de la compañía de streaming son especialmente evangelizadores de las bondades de la misma, por lo que se busca aprovechar su potencial, reforzar su opinión, que suele ser fundada, apasionada y cualificada, lo que les convierte en excelentes prescriptores.

Es importante señalar que para las compañías los early adopters tienen preferencia por los deseos y aspiraciones, y no tanto en las necesidades reales, o en general no son su principal motivación. Por todo ello se convierten en un elemento clave de una estrategia comunicativa innovadora y que coadyuve en la expansión de la compañía. Así, tanto a nivel internacional como si observamos un país como España, en pocas horas, la etiqueta \#RenewSense8 se coloca como trending topic, siendo tendencia durante varias horas, tal y como refleja en el Gráfico 3, que relaciona el tiempo y los miles de mensajes: 


\section{Gráfico 3}

Evolución del hashtag \#RenewSense8

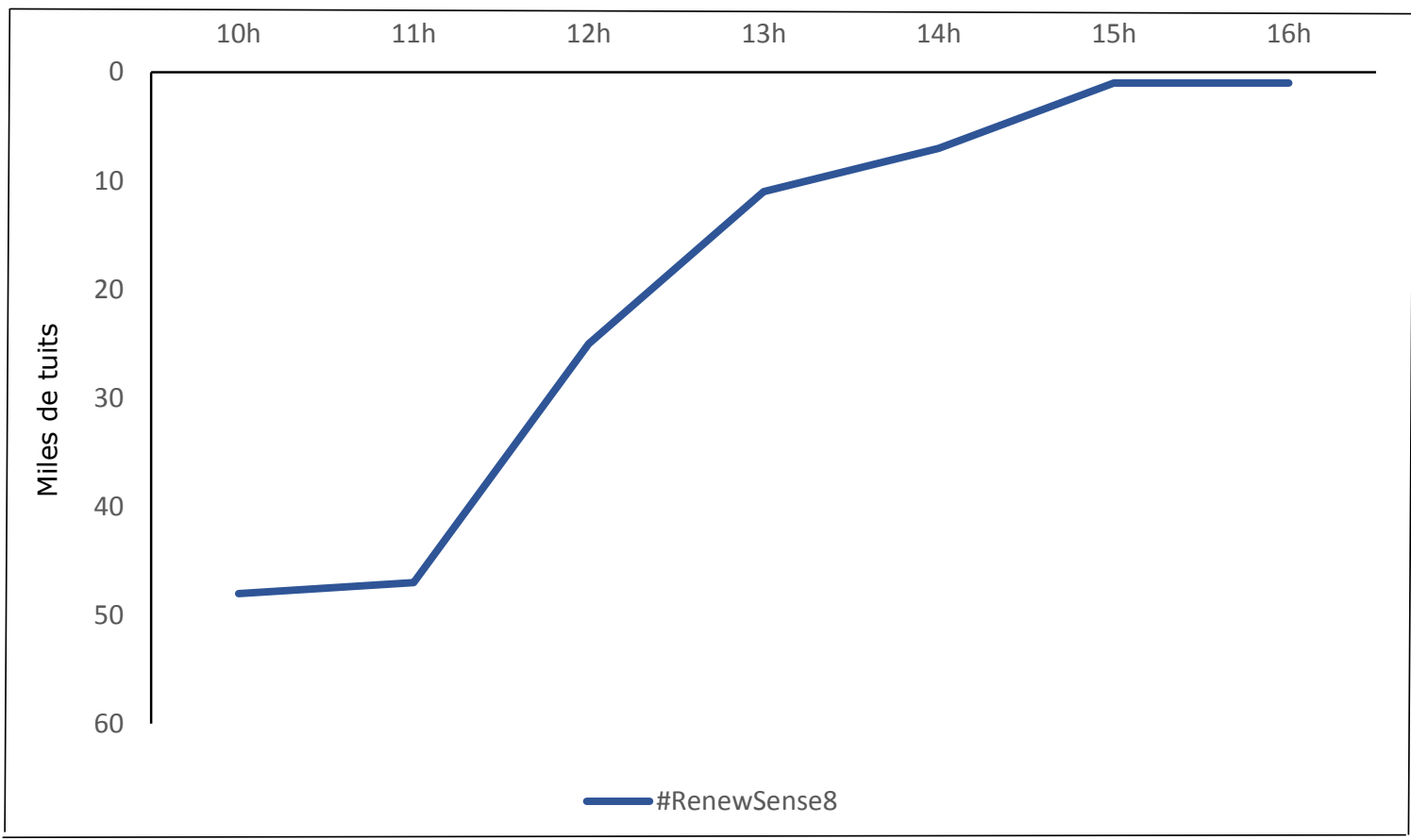

Fuente: Trendinalia España (2 jun. 2017).

Este fenómeno mundial viene acompañado de un problema muy significativo para Netflix, y es que, son muchos los fans de la serie que amenazan con abandonar la plataforma, extendiendo una corriente de opinión precisamente en esos suscriptores denominados primeros adoptantes, sector clave, como se ha señalado, en la expansión internacional.

A continuación (véase Ilustraciones 1 y 2), y a modo de ejemplo de las reacciones suscitadas por la comunidad, se muestran algunos de los tuits que se han señalado. Como se ha advertido, no solo se expresa el descontento por la cancelación de la serie, sino que, adicionalmente, se amenaza con darse de baja de la propia plataforma, e incluso, con irse a la competencia, como señal de provocación; una elevada y ruidosa protesta en redes y comunidades virtuales que generó un estado de opinión adverso en un momento clave de la expansión de la plataforma. 


\section{Ilustración 1}

\section{Tuits de fans tras la cancelación oficial de Sense8}

\section{\#SNSD\&IZ*ONE}

@Danna_1516

\#sense8 es una de las MEJORES series que he visto, valía la pena mi membresía...I don't need @netflix anymore. BYE

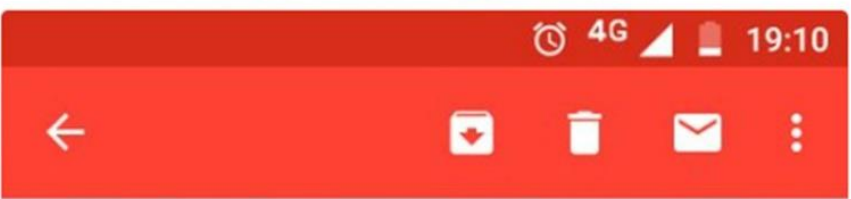

\section{NETFLIX}

\section{Tu confirmación de cancelación}

Hola, Dayana:

Tal rnmn In colisitacto

○ 483 2:14 - 2 jun. 2017

218 personas están hablando de esto

Fuente: Twitter, 2 jun. 2017.

Es aquí, donde empieza la verdadera situación de crisis comunicativa de la empresa. En este sentido, y a través de la plataforma Change.org, los fans de la serie recogieron un total de 524.727 firmas para conseguir que la productora audiovisual diese marcha atrás, y, simultáneamente, darse de baja de Netflix. 
Ilustración 2

Imagen de la petición de los fans en Change.org

\begin{tabular}{|c|c|c|c|}
\hline change.org Inicia una petición Mis peticiones Más peticiones & Programa de socios & Entrar \\
SENSE 8 SEASON 3 RENEWAL & Victoria confirmada \\
gracias a 524.727 firmas!
\end{tabular}

Fuente: Twitter, 4 jul. 2017.

No obstante, la decepción del público no tardó en llenar titulares de diversos medios y plataformas de comunicación especializadas. Por lo que, a la ya problemática de perder un porcentaje de suscriptores muy elevado, se le unía el problema de hacer frente a toda una campaña sin precedentes de mala imagen para la compañía, que encierra, a modo de ejemplo, los siguientes titulares de prensa generalista española: "Netflix cancela Sense8... y Twitter carga contra ella" - Huffpost (Poncel, 2017); "Casi medio millón de usuarios de Netflix amenazan con darse de baja tras la reciente cancelación de Sense8" $(A B C, 2017)$; o también "Netflix deja los suscriptores a medias al cancelar Sense8 sin un final" - La Vanguardia (Solá Gimferrer, 2017); éstos son solo algunos de los muchos titulares que días después del anuncio oficial de la cancelación de la serie, aparecían en los medios de comunicación de España. 
Como se puede apreciar, la campaña que comenzó en redes sociales evolucionó en un fenómeno comunicativo que transcendía las barreras nacionales y creaba una serie de dudas sobre la reputación de la plataforma Netflix en un público virtual imprescindible en su difusión joven y urbana: los early adopters.

La plataforma, envuelta en una crisis de imagen, intenta apelar a la lógica de los usuarios mediante un comunicado donde expresa los motivos por los que la serie ha sido cancelada. Para ello, elige la red social Tumblr, en espacio de microblogging oficial de la serie, y Facebook, para dar a conocer el siguiente comunicado:

Para nuestra familia Sense8 (...). Hemos visto las peticiones. Hemos leído los mensajes. Sabemos que quiere \#RenewSense8, y deseamos poder \#BringBackSense8 por usted. El motivo por el que nos hemos tardado tanto en responderte es porque hemos pensado mucho en Netflix para intentar que funcione, pero desafortunadamente no podemos. Gracias por mirar y espero que te mantengas cerca de tu clúster en todo el mundo. \#SensatesForever (Facebook, Sense8, 8 jun. 2017).

No obstante, y además de este breve comunicado, el director de contenidos de la plataforma, Ted Sarandos, explicó que se trataba de un problema mucho más básico. En este sentido, Sarandos detalló que se debía a unos costes muy elevados de producción, alrededor de 9 millones de dólares por episodio, y una audiencia poco elevada (EI Mundo, 2017).

Estas explicaciones no fueron suficientes para la comunidad de fans de la serie, organizada en opinión pública virtual, que seguía aumentando en sus esfuerzos de lograr que la plataforma cerrará de algún modo Sense8. De este modo, el número de firmas en la plataforma Change.org no dejó de aumentar. La presión en Twitter se mantenía, sobre todo, a través de los hashtags \#RenewSense8 y \#BringBackSense8.

Sin embargo, las diversas explicaciones no consiguieron calmar a los usuarios en redes sociales, que seguían intentando hacer que su serie continuase. Las firmas en la plataforma Change.org superaron el medio millón, cuando, a través del perfil oficial de la serie en Twitter, el 29 de junio de 2017, Lana Wachwoski, co-creadora de la serie, anunciaba que ésta tendría un capítulo final de dos horas de duración y mostraba un texto que anticipaba la nueva y sorprendente estrategia comunicativa de Netflix: sumarse a la misma corriente de opinión pública como una parte más de la misma, como se reproduce en el siguiente Ilustración 3, y que supuso un repunte en la imagen de la plataforma. Esta nueva estrategia nos introduce de lleno en una nueva forma de entender la comunicación entre la marca y el usuario, siendo la propia compañía parte fundamental del propio mensaje, y uniéndose a las reclamaciones de los propios usuarios como si fueran parte de la corriente. 


\section{Ilustración 3}

\section{Lana Wachwoski en Twitter y nueva estrategia de la compañía}

Dear Sense8 Family,

I have been meaning to write this letter for some time. The outpouring of love and grief that came in the wake of the news that Sense8 would not be continuing was so intense that 1 often found myself unable to open my own email. I confess I fell into a fairly serious depression.

I had never worked so hard, or put so much of myself into a project as I had with Sense8 and its cancellation hollowed me out. I felt the disappointment of my amazing crew (I wish people could understand the impossibilities they achieved with implausible regularity.) I felt the sadness of the actors who had given so much of themselves, always finding more whenever the sun broke from the clouds. But most of all I felt the heartbreak of our fans (again I wish I could cluster with you to share some of the beautiful moments, the hugs, tears and laughter, as well as the insightful and humbling conversations I have had with people who have connected to this show, they are unlike any fans I have ever encountered as an artist).

Friends kept calling from all over the world asking, 'Isn't there anything you can do?*

And the truth was, no. By myself, there was nothing I could do.

But just as the characters in our show discover that they are not alone, I too have learned that I am not just a me. I am also a we.

The passionate letters, the petitions, the collective voice that rose up like the fist of Sun to fight for this show was beyond what anyone was expecting.

In this world it is easy to believe that you cannot make a difference; that when a government or an institution or corporation makes a decision, there is something irrevocable about the decision; that love is always less important than the bottom line.

But here is a gift from the fans of this show that I will carry forever in my heart: while it is often true those decisions are irreversible, it is not always true.

Improbably, unforeseeably, your love has brought SenseB back to life. (I could kiss every single one of youl)

It is my great pleasure as well as Netfix's (believe me, they love the show as much as we do but the numbers have always been challenging) to announce that there will be another two hour special released next year. After that... if this experience has taught me anything, you NEVER know.

Thank you all. Now let's go find out what happens to Wolfgang.

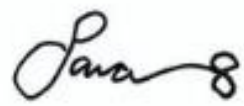

\section{(9) Sense8}

@sense8

Death doesn't let you say goodbye. 2 hour finale episode in the works. Tell your cluster.

20:39 - 29 jun. 2017

\section{6,8 mil $Q 68,9$ mil personas están hablando de esto}

Fuente: Twitter, 29 jun. 2017. 
Las redes sociales han demostrado en numerosas ocasiones como pueden ser generadoras de opinión pública y pueden servir de altavoz para las comunidades. En este estudio de caso, sirvió como herramienta de interacción comunicativa para que los seguidores de la serie de ficción Sense8 de la plataforma Netflix lograrán que ésta produjese un episodio final que cerrara la línea argumental de la misma. Si bien el proceso de cancelación y anuncio de un episodio final ha demostrado la fuerza de las redes sociales, también ha hecho que Netflix afrontara una de sus crisis más fuertes hasta la fecha. Es el modo en el que ha resuelto la crisis donde radica lo verdaderamente relevante a nuestro juicio de este estudio.

Por su parte, la compañía llevó a cabo una triple estrategia para contrarrestar una opinión pública especializada adversa. Por un lado, abordándola desde el punto de vista empresarial y de producción. Si bien, los costes eran muy elevados para la "rentabilidad" de la serie, como hemos señalado, Netflix asumió que quería llevar a cabo un final que pusiera fin a la mala imagen que la plataforma estaba adquiriendo, así puso en marcha todo lo relativo a contactos con actores, guionistas y el anuncio final de la realización de un nuevo episodio.

Pero, lo más relevante en este punto es la puesta en marcha de una estrategia comunicativa donde la propia empresa, a través de sus cuentas, se suma a las protestas dando a entender que los fans de la serie no están fuera de la compañía, sino que las reivindicaciones también forman parte de los propios trabajadores de la compañía. Este primer aspecto es innovador y ciertamente descarado o insólito, al desvincularse la empresa en su comunicación mediática a su propia decisión empresarial, participando de la corriente de opinión de los fans sobre el regreso de la serie; todo ello no se produce de una manera conflictiva, sino como forma parte de la misma comunidad afectada que los seguidores.

En segundo lugar, y de forma coordinada y simétrica, se suceden los comunicados en Twitter de los propios creadores y miembros de la serie. Para ello, lo realmente singular de este segundo aspecto, y que demuestra la coordinación comunicativa empresarial, es que en la mayoría de los casos en los textos los miembros del equipo hacen partícipe a la compañía del éxito de la creación de un episodio final. Este hecho se aprecia con facilidad en el comunicado de Lana Wachwoski, co-creadora de la serie, quien afirma en su texto de anuncio en Twitter (Ilustración 3) que "es un gran placer para mí y para Netflix (créanme, ellos aman el show tal como nosotros, pero los números siempre han sido un desafío) anunciar que habrá un nuevo especial de dos horas que se estrenará el próximo año".

En definitiva, este singular estudio de caso pone de manifiesto la fuerza de la opinión pública mediática especializada, así como el uso de nuevas estrategias comunicativas para liderarla o contrarrestarla. La crisis de confianza e identificación de sus audiencias con la compañía Netflix en este caso se cierra o al menos se reconduce al desarrollarse una estrategia comunicativa triple, ejecutada por la propia compañía, 
coordinando comunicativamente los mensajes en redes virtuales (singularmente Twitter), la dirección y producción de la serie y los actores con los que el público empatiza, para interactuar en el mismo sentido, aunar el espíritu global en función de un objetivo compartido, diluir la confrontación y cerrar finalmente este primer episodio de crisis del operador audiovisual.

\section{Conclusiones}

Del estudio de caso analizado se desprende una primera conclusión general que define cómo la opinión pública y las corrientes comunicativas de los seguidores de productos audiovisuales cobran relevante importancia para las compañías, especialmente en el sector de la nueva economía y la nueva televisión. Los nuevos operadores audiovisuales (Netflix, HBO, Amazon Prime Video etc.), que operan en streaming, ofreciendo contenidos altamente personalizables y bajo demanda, están rompiendo la hegemonía de la industria audiovisual y televisiva tradicional.

A su vez, dentro de estas corrientes de opinión, difundidas a través de redes virtuales, los primeros adoptantes (early adopters) resultan de una especial relevancia para estas compañías, al ser un público cualificado, pasional y evangelizador de las bondades de la empresa y son especialmente cuidados en la estrategia a largo plazo de expansión internacional de operadores como Netflix. Para generar o difundir opinión pública favorable, estas nuevas empresas audiovisuales están utilizando estrategias comunicativas singulares y novedosas para lograr su expansión internacional, donde el papel de las campañas en redes sociales, la viralización de contenidos y la interactividad con los usuarios llevan o toman un paso por delante a los operadores televisivos tradicionales.

Como ejemplo paradigmático, Netflix basó su inicial estrategia de expansión internacional en la generación de contenidos originales, rompedores y dirigidos a comunidades segmentadas, pero globales, apostando por costosas producciones firmadas o producidas por creadores audiovisuales contemporáneos reconocidos. Este es el caso de la serie Sense8, dirigida por las hermanas Wachowski, cuyos contenidos incluyen la utilización de formas novedosas y singulares de apelación a los sentimientos, a la espiritualidad o a la identidad sexual. La serie es un ejemplo de estrategia de fidelización de nuevas audiencias en su expansión internacional. Por el mismo motivo su cancelación debido a los altos costes de producción supuso un primer episodio de crisis con sus públicos suscriptores.

El caso estudiado es muy singular dado que se trató de una primera crisis comunicativa de estos nuevos operadores de contenido audiovisual, caso concreto de Netflix, y se produce a nivel mundial debido a la propia idiosincrasia de la serie. Esto conlleva una quiebra de la estrategia de implicación y apelación a las audiencias, mantenida comercialmente mediante la suscripción al canal. Desde el punto de vista 
empresarial, Netflix asumió que quería llevar a cabo un final que pusiera fin a la situación de crisis de imagen de marca que la plataforma estaba adquiriendo, por parte de un público singular seguidor de la serie, no muy numeroso pero influyente, así puso en marcha una triple estrategia que involucra contactos con actores, guionistas, el público y el anuncio final de la realización de un nuevo episodio.

Del análisis del contenido de la red social Twitter se desprende que la compañía desarrolló una estrategia comunicativa innovadora donde la propia empresa se suma a las protestas de su audiencia en contradicción con su misma decisión empresarial, dando a entender que los fans de la serie no están fuera de la compañía, sino que las reivindicaciones también forman parte de los propios trabajadores de la compañía. Esto es, no es un agente externo, sino que forma parte de la misma comunidad afectada que los seguidores.

Se observa igualmente como abundan los comunicados en Twitter de los propios creadores y miembros de la serie. Pero, lo realmente destacable de este segundo aspecto es que, en la mayoría de los casos, los miembros del equipo hacen partícipe a la compañía del éxito de la creación de un episodio final.

Se produce una estrategia de diversificación comunicativa en la esfera virtual (esencialmente en la red social Twitter) donde opera la corriente de opinión crítica, que incluye a los comunicados de la compañía, el elenco creativo de la serie (dirección, producción ejecutiva y actores) y que culmina con el anuncio de la reanudación de la producción. Es decir, se supera el tradicional concepto de prosumidor audiovisual, como señala, por ejemplo, Ferreras-Rodríguez (2014), dado que estas comunidades virtuales no solo generan contenido, sino que producen decisiones estratégicas. Este artículo amplía el grado de respuesta de las comunidades virtuales contemporáneas de otros estudios como los de González-Neira y Quintas-Froufe (2016), que encontraron menor grado de conexión con las decisiones empresariales. Otros estudios de referencia sobre Nexflix, como el de Izquierdo-Castillo (2015), se refieren al nuevo negocio mediático liderado por la compañía con escasa consideración, más allá del modelo de uso técnico, de las comunidades en redes sociales. Por otra parte, y respecto al contenido de la serie analizada, pese que Anaz (2017) habla de una representación estereotipada de la identidad personal moderna, lo cierto es que la serie logró una profunda conexión emocional con generadores de opinión en la red, y su activismo en el proceso se hizo muy notorio. Un estudio que advertía de la creciente relación simbiótica entre Twitter y la televisión es el de Wang (2016); en ese mismo sentido las conclusiones de este estudio están también en la línea de trabajos anteriores como los de Greer y Ferguson (2011) o Harrington, Hihfield y Bruns (2013), que anticiparon certeramente el poder de Twitter y las audiencias activas como red influyente en la empresa audiovisual. En nuestro estudio de caso, Netflix tuvo al final que asumir la corriente de público desafecto y cerrar este episodio de crisis comunicativa y de imagen de la compañía. 
Finalmente, este estudio de caso debe ser completado en el futuro con otros ejemplos de situación comunicativa e interacción con seguidores y suscriptores de otras empresas innovadoras, para comprobar hasta qué punto son utilizadas estas nuevas estrategias para modelar e influir en la opinión pública especializada respecto a ellas.

\section{Referencias bibliográficas}

$A B C$. "Casi medio millón de usuarios de Netflix amenazan con darse de baja tras la reciente cancelación de Sense8", 6 jun. 2017. Disponible en: <https://www.abc.es/play/series/noticias/abci-usuariosnetflix-amenazan-darse-baja-tras-reciente-cancelacion-sense8-201706061415_noticia.html>. Acceso en: 7 dic. 2017.

ADHIKARI, V., et al. "Measurement study of Netflix, Hulu, and a tale of three CDNs". IEEE/ACM Transactions on Networking (TON), vol. 26, p. 1.984-1.997, 2015.

AIMC - Asociación para la Investigación de Medios de Comunicación. "Estudio general de medios". Disponible en: <http://reporting.aimc.es/index.html\#/main/cockpit>. Acceso en: 5 feb. 2018.

ANAZ, S. "O arcaico e o contemporâneo em Sense8: representações arquetípicas da diversidade". Intexto, vol. 40, p. 77-95, 2017.

BEAUMONT, J. F. "Digitalización, convergencia, multimedia. Claves del nuevo escenario audiovisual español". Cuenta y Razón, vol. 117, p. 37-46, 2000.

Bernal Triviño, A. I. Los nuevos medios de comunicación y los jóvenes: aproximación a un modelo ideal de medio. Madrid: EuroEditions, 2009.

CAMPos-Freire, F. "El futuro de la TV europea es híbrido, convergente y cada vez menos público". Revista Latina de Comunicación Social, vol. 68, p. 89-118, 2013.

. "Adaptación de los medios tradicionales a la innovación de los metamedios". El Profesional de la Información, vol. 24, no 4, p. 441-450, 2015.

CASTElló MARTínez, A. "El uso de hashtags en Twitter por parte de los programas de televisión españoles". In: I Congreso Internacional Comunicación y Sociedad. Logroño, 2013.

Claes, F.; Deltell, L. "Audiencia social en Twitter: hacia un nuevo modelo de consumo televisivo". Trípodos, vol. 36, p. 111-132, 2015.

CNMC - Comisión Nacional de los Mercados y la Competencia. "Qué es la CNMC", 2017. Disponible en: <https://www.cnmc.es/sobre-la-cnmc/que-es-la-cnmc>. Acceso en: 16 feb. 2018.

DAHLGREN, P. Paisaje mediático cambiante y participación política. In: MoRAGAS, M. La comunicación: de los orígenes a internet. Barcelona: Gedisa, 2012.

Dawson, M. "Television between analog and digital". Journal of Popular Film \& Television, vol. 38, no 2, p. 95-100, 2010.

EL PAÍs. "Netflix cancela Sense8 tras dos temporadas", 2 jun. 2017. Disponible en: <https://elpais.com/cultura/2017/06/01/television/1496340078_798311.html>. Acceso en: 8 jul. 2017.

EL MUNDO. "Netflix explica la cancelación de Sense8 y The Get Down", 2017. Disponible en: <https://happyfm.es/musica/netflix-explica-la-cancelacion-de-sense8-y-the-get-down-51264/>. Acceso en: 20 jun. 2017. 
Ferreras-Rodríguez, M. "Los nuevos prosumidores: audiencias de la televisión social. Análisis de Operación Palace en Twitter". Revista Mediterránea de Comunicación, vol. 5, nº 1, p. 175-192, 2014.

Fundación TelefónicA. La sociedad digital en España. Madrid: Fundación Telefónica, 2017.

GALLEGo, F. "Social TV analytics: nuevas métricas para una nueva forma de ver televisión". Index.Comunicación, vol. 3, p. 13-39, 2013.

GILLAN, J. Television and new media. New York: Routledge, 2011.

Gómez-Domínguez, P. "Era digital y televisión autonómica: un estudio comparativo de las plataformas $w e b$, aplicaciones móviles y redes sociales de TV3 y BBC One". Communication \& Society, p. 85-116, 2016.

GómEZ-URIBE, C.; HUNT, N. "The Netflix recommender system: algorithms, business value, and innovation". ACM Transactions on Management Information Systems, vol. 6, no 4, 2015.

GonzÁlez-NeirA, A.; QuintAS-Froufe, N. "El comportamiento de la audiencia lineal, social y en diferido de las series de ficción españolas". Revista de la Asociación Española de Investigación de la Comunicación, vol. 3, no 6, p. 27-33, 2016.

Greer, C.; Ferguson, D. "Using Twitter for promotion and branding: a content analysis of local television Twitter sites". Journal of Broadcasting \& Electronic Media, vol. 55, no 2, p. 198-214, 2011.

HABERMAS, J. Öffentlichkeit (ein Lexikonartikel). Francfort a.M.: Suhrkamp, [1964] 1973.

Harrington, S.; Hihfield, T.; BRuns, A. "More than a backchannel: Twitter and television". Participations Journal of Audience \& Reception Studies, vol. 10, no 1, p. 405-409, 2013.

Heredia Ruiz, V. "Revolución Netflix: desafíos para la industria audiovisual". Chasqui, vol. 135, p. 275-295, 2017.

Highfield, T.; HARRington, S.; BRUnS, A. "Twitter as a technology for audiencing and fandom". Information, Communication \& Society, vol. 16, no 3, p. 315-339, 2013.

HIPERTEXTUAL. "Netflix explica por qué cancela Sense8", 9 jun. 2017. Disponible en: <https://hipertextual.com/2017/06/netflix-sense8-cancelacion-motivos>. Acceso en: 30 ene. 2018.

IAB-Spain. Estudio Anual de Redes Sociales, 2017.

INFORME CONECTA. La televisión de pago en España. Madrid: Deloitte, 2018.

IzQUiERDo-CASTILlo, J. "El nuevo negocio mediático liderado por Netflix: estudio del modelo y proyección en el mercado español". El Profesional de la Información, vol. 24, n 6, p. 819-826, 2015.

JeNKINS, H. "Transmedia storytelling: moving characters from books to films to video games can make them stronger and more compelling". MIT Technology Review, jan. 2003. Disponible en: <http://www.technologyreview.com/news/401760/transmedia-storytelling>. Acceso en: 18 ago. 2018.

JENKINS, H.; Ford, S.; GREEN, J. Spreadable media creating value and meaning in a networked culture. New York: New York University Press, 2013.

JenNer, M. "Is this TVIV? On Netflix, TVIII and binge-watching". New Media \& Society, vol. 18, no 2, p. 257-273, 2016.

KeEgan, C. M. "Tongues without bodies: the Wachowskis' Sense8". TSQ - Transgender Studies Quarterly, vol. 3, no 3-4, p. 605-610, 2016. 
LACALLE, C. "La ficción interactiva: televisión y web 2.0. Ámbitos. Revista Internacional de Comunicación, no 20, p. 87-107, 2011.

LipPMANN, W. La opinión pública. Madrid: Cuadernos de Langre, 2003.

López Vidales, N.; González Aldea, P.; Medina de la Villa, E. "Jóvenes y televisión en 2010: un cambio de hábitos". Zer, vol. 16, nº 30, p. 97-113, 2011.

McComBs, M. Influencia de las noticias sobre nuestras imágenes del mundo. Barcelona: Paidós, 1996.

McComBS, M. E.; SHAw, D. "The agenda-setting function of mass media". Public Opinion Quartely, vol. 36, nº 2, p. 176-187, 1972.

MONTOYA, E. "Diseño e implementación de una videoteca digital". En: III Congreso Iberoamericano de Telemática Aplicada, 2003.

PONCEL, M. "Netflix cancela Sense8... y Twitter carga contra ella". Huffpost, 2 jun. 2017. Disponible en: <https://www.huffingtonpost.es/2017/06/02/netflix-cancela-sense8-y-twitter-carga-contraella_a_22122347>. Acceso en: 7 dic. 2017.

QuinTAS-Froufe, N.; GonZález-Neira, A. "Audiencias activas: participación de la audiencia social en la televisión". Comunicar, vol. 43, p. 83-90, 2014.

RAYMOND, J. "The newspaper, public opinion, and the public sphere in the seventeenth century". Prose Studies: History, Theory, Criticism, vol. 2, n 21, p. 109-136, 1998.

Rodríguez Rosell, M., Melgarejo Moreno, I. "El educomunicador: un profesional necesario en la sociedad multipantalla". Sphera Publica, no 12, p. 143-159, 2012.

RUANo, S. "Internet y la telefonía móvil nuevos soportes para distribuir contenidos audiovisuales". Razón y Palabra, vol. 68, 2009.

RUBio FeRRERES, J. M. "Opinión pública y medios de comunicación. Teoría de la agenda setting". Gazeta de Antropología, vol. 25, no 1, 2009. Disponible en:

<https://www.ugr.es/ pwlac/G25_01JoseMaria_Rubio_Ferreres.html>. Acceso en: 15 ene. 2018.

Ruiz del Olmo, F. J.; BeLmonte JimÉnez, A. M. "Los jóvenes como usuarios de aplicaciones de marca en dispositivos móviles". Comunicar, no 43, p. 73-81, 2014.

SolÁ Gimferrer, P. "Netflix deja los suscriptores a medias al cancelar Sense8 sin un final". La Vanguardia, 5 jul. 2017. Disponible en:

<http://www.lavanguardia.com/series/20170602/423138580266/netflix-cancela-sense8-dejasuscriptores-a-medias.html> Acceso en: 2 feb. 2018.

THE HOLLYWOOD REPORTER. "Sense8 canceled at Netflix", 1 jun. 2017. Disponible en: https://www.hollywoodreporter.com/live-feed/sense8-canceled-at-netflix-1009404. Acceso en: 12 ene. 2018.

TRENDINALIA ESPAÑA. 2 jun. 2017. Disponible en: <http://www.trendinalia.com/twitter-trendingtopics/spain/spain-today.html>. Acceso en: 25 jul. 2020.

Valenzuela, S.; Puente, S.; Flores, P. "Comparing disaster news on Twitter and television: an intermedia agenda setting perspective". Journal of Broadcasting \& Electronic Media, vol. 61, no 4, p. 615-637, 2017.

WANG, Y. "How do television networks use Twitter? Exploring the relationship between Twitter use and television ratings". Southern Communication Journal, vol. 81, nº 3, p. 125-135, 2016. 
OPINIÓN PÚBLICA Y NUEVAS ESTRATEGIAS COMUNICATIVAS EN LA INDUSTRIA AUDIOVISUAL

WoHN, Y.; NA, E.-K. "Tweeting about TV: sharing television viewing experiences via social media message streams". First Monday, vol. 16, 2011. Disponible en:

<https://firstmonday.org/article/view/3368/2779>. Acceso en: 20 dic. 2017.

Woods, F. British youth television: transnational teens, industry, genre. London: Palgrave Macmillan, 2016.

Wu, T. "Netflix contra la cultura de masas". Letras Libres, vol. 16, no 182, p. 10-15, 2014.

YIN, R. K. Case study research: design and methods. 4th ed. Thousand Oaks, CA: Sage, 2009.

\begin{abstract}
Public opinion and new communication strategies in the audiovisual industry: the case of Netflix and Sense8

Streaming audiovisual operators are breaking the contemporary audiovisual ecosystem with a growing and majority influence in urban young people, firstly, to constitute a serious competition for traditional television channels. In addition to this, social networks and the interactivity that audiovisual contents provide with audiences are also a paradigm change in public activity. In this context, this article analyzes the situation of public opinion and communicative crisis that occurred from the cancellation of content (the Sense8 series of Netflix, 2017) and the impact on social networks (Twitter). Among its conclusions is a novel strategy by this new audiovisual company to respond and integrate in their discourse and productive practices the demands of their audiences.
\end{abstract}

Keywords: communication in crisis; audiovisual operators; Netflix; Twitter; Sense8

\title{
Resumo
}

Opinião pública e novas estratégias de comunicação na indústria audiovisual: o caso da Netflix e da série Sense8

Os operadores audiovisuais de streaming estão rompendo o ecossistema audiovisual contemporâneo com uma influência crescente e predominante nos públicos jovens urbanos, em primeiro lugar, para constituir uma concorrência séria aos canais de televisão tradicionais. Junto a isso, as redes sociais e a interatividade que os conteúdos audiovisuais proporcionam às plateias são também uma mudança de paradigma na atividade do público televisivo. Nesse contexto, o presente artigo analisa, por meio de um estudo de caso, a situação de crise comunicativa que surgiu a partir do cancelamento de conteúdos (a série Sense8, 2017, produzida pela Netflix) e a repercussão em redes sociais (Twitter). Entre suas conclusões, aponta-se uma nova estratégia por parte desse tipo de empresa audiovisual para responder às demandas de seus públicos e integrá-las em seu discurso e práticas produtivas. Palavras-chave: comunicação em crise; operadoras de audiovisual; Netflix; Twitter; Sense8

\section{Résumé}

L'opinion publique et les nouvelles stratégies de communication dans l'industrie audiovisuelle: le cas de Netflix et de la série Sense8

Les opérateurs audiovisuels en streaming brisent l'écosystème audiovisuel contemporain avec une influence croissante et prédominante sur les jeunes publics urbains, en premier lieu, pour constituer une concurrence sérieuse aux chaînes de télévision traditionnelles. Parallèlement à cela, les réseaux sociaux et l'interactivité que les contenus audiovisuels fournissent aux publics constituent également un changement de paradigme dans l'activité du public de la télévision. Dans ce contexte, le présent travail analyse, à travers une étude de cas, la situation de crise communicative issue de l'annulation de contenus (la série Sense8, 2017, produite par Netflix) et la répercussion sur les réseaux sociaux (Twitter). Parmi ses conclusions, il vise une nouvelle stratégie de la part de cette nouvelle société 
audiovisuelle pour répondre et intégrer dans ses discours et ses pratiques productives les demandes de ses publics.

Mots-clés: communication en crise; opérateurs audiovisuels; Netflix; Twitter; Sense8

Artigo submetido à publicação em 7 de agosto de 2018 . Artigo ressubmetido à publicação em 27 de julho de 2019.

Versão final aprovada em 13 de julho de 2020.

Opinião Pública adota a licença Creative Commons CC-BY. 